\title{
Characterization and reduction of exercise-based motion influence on heart rate variability using accelerator signals and channel decoding in the time-frequency domain
}

\author{
Iman Alikhani, Kai Noponen, Arto Hautala and Tapio Seppänen *
}

September 13, 2019

\begin{abstract}
Heart rate variability (HRV) is defined as the variation of heart's beat to beat time intervals. Although HRV has been studied for decades, its response to stress tests and off-rest measurements is still open to controversy. In this paper, we studied the influence of motion on HRV throughout different exercise tests, including a maximal running of healthy recreational runners, cycling and walking tests of healthy subjects. In our proposed method, we utilize the motion trajectory (which is known to exist partially in HRV) measured by a three-channel accelerator (ACC) and estimate their share in HRV, derived from a wearable electrocardiography (ECG), in an error correcting problem formulation. In this method, we characterize the motion components of three orthogonal directions induced into the HRV signal and then, we suppress the estimated motion artifact to construct a motion-attenuated spectrogram. Our analysis shows that HRV in exercise context is susceptible to motion artifacts and the interpretation of autonomic nervous system (ANS) activity and HRV indexes throughout exercise is of high error margin, depending on the intensity level, type of exercise and motion trajectory. Our experiment on 84 healthy subjects throughout a mid-intensity cycling and walking tests show 39 and $32 \%$ of influence in average, respectively. In addition, our proposed method investigated through a maximal running test on 11 runners reveals that motion can describe in average 20 to $40 \%$ of the HRV high frequency (HF) energy at different workloads of running.
\end{abstract}

\section{Introduction}

As many body organs, heart is regulated by the nervous system and heart rate variability (HRV) is an indicator of autonomic nervous system (ANS) activity and cardiovascular system functionality [6]. The response of ANS activity to different external and internal stimuli is reflected by the HRV which is the beat-to-beat timing variation of cardiac electrical activity [21]. The variability of inter-beat intervals (IBI) as a non-invasive biosignal has been investigated for years in diagnosis and clinical studies [16, 8, 15, 11], sport physiology [24, 25, 22], and many other disciplines. Consequently, there are numerous standards, review and surveys in the literature regarding the extraction, preprocessing, analysis methodology and interpretation of HRV $[6,1,24]$.

The HRV literature substantially contributed to controlled resting state studies and hence, the principles are primarily established in such contexts. In the past, the practical complications, as well as the high-price of HRV tracking outside of the clinical environment was probably a reason of less investigations in daily-life context. More recently, by the rise of low-cost wearable technologies and the convenience of heart activity monitoring in versatile configurations (e.g. by electrocardiography (ECG) chest straps), the number of studies of cardiac performance in response to different physical stressors has increased (e.g ANS response to a maximal or sub-maximal running test as a physical stimulus). In some cases, studies in these contexts has led to modification and adaption of the established standards, such as redefinition of the HRV high-frequency (HF) component's range during physical exercise in $[3,21]$.

*Affiliation: Physiological Signal Analysis Team, Center for Machine Vision and Signal Analysis, University of Oulu, Oulu, Finland, Email: firstname.lastname@oulu.fi 
Wearable technologies offer new means of biomarker tracking to sport physiologists and rehabilitation experts, who are exploring the responses of ANS and cardiac system to different stressors. It is generally known that under increasing exercise intensity, the baroflex effect is suppressed along with the increasing heart rate leading to decrease in HRV. On the other hand, increasing motion will also have a rising influence on the observed HRV. However, the actual proportion of motion artifacts is still not very well-investigated. One major source of HRV contamination is known to be cadence [5, 3]. Recently, the study of HRV during stress test has been of interest $[2,3,13,14,17,18]$. In 2009, Blain et al. in [5] revealed the existence of cardiolocomotion coupling (CLC) components in HRV during cycling. They observed a HF rising energy band associated with the pedaling frequency in the HRV spectral content. They also state that the corresponding energy of pedaling frequency in the HRV spectrogram increases with the exercise workload. Similarly, Bailon et al. in [3] observed component associated with the stride frequency (SF) during a running protocol. They claim that the SF and its second harmonic are predominantly influencing the HRV dynamic and introduce new components into its spectral contents, which accounts for approximately $22 \%$ of the HRV spectral energy. However they claim that the associated SF power does not increase by the rise of exercise intensity. To avoid any misinterpretation of HRV during running, those should be taken into account as SF components overlap with the HF and low frequency (LF) components of HRV. A similar phenomenon was found in a cross-country skiing experiment in [20], where they identified components linked with the poling frequency in cross-country skiing's HRV spectrogram.

Despite the aforementioned, many HRV studies during both sub-maximal and maximal physical stress tests have been conducted in the past but also recently overlooking the motion artifacts and their substantial influence on the interpretation of HRV indexes, e.g. in $[9,10,26]$ among others. Moreover, the recently published review paper [21] states that there is inconsistency in the response of HRV spectral indexes to exercise intensity. Despite the methodological diversity of HRV analysis declared in [21] as a possible cause, we hypothesize that a major origin of the inconsistency is the influence of motion artifacts on the spectral content of HRV, which leads to misinterpretation of autonomic nervous response to exercise intensity. Recently, the paper [13] published by Hernando et al., offers a methodological framework in order to characterize the CLC components in HRV spectrogram, experimented on cycling and running protocols. They reported that CLC components account for $10 \%$ of the total spectral power at the beginning of the exercise and up to $30 \%$ near maximal heart rate exceeding the anaerobic threshold (AT). Clearly, there is a need for methods to address the external elements (e.g. motion-caused disturbances) affecting HRV in stress test contexts due to the major influences they potentially have on computations and interpretations. Yet, the duration, intensity and modality of the exercise protocol as well as subject's performance and fitness level might elicit different effects on HRV [21]. Thus, the current proposed models such as [13,3] may not be comprehensive enough to provide a general solution to the problem, and it is likely that more flexible models could provide better context sensitivity and accommodation to the aforementioned factors.

In this paper, we study the influence of motion on HRV using inertial sensoring in cycling, walking and maximal running exercise tests. Contrary to the literature [3, 5, 13], our analysis of motion is not founded on the cadence or pedaling frequency. Instead, we aim for a more holistic description of the movement by contrasting the perceived movements of a threechannel microelectromechanical systems (MEMS) accelerator (ACC) on the subjects' chests with simultaneously recorded ECG signal. In the analysis we comprehensively characterize the effects of motion and their sizes in the power spectral domain.

\section{Material and Method}

In this section we describe the empirical data and methods used in this study. First, we describe the database and the underlying measurement protocol in sub-section 2.1. Second, the preprocessing steps - including ECG preprocessing, R-wave annotation, construction of IBI, analysis of ACC data - are outlined in 2.2. Third, in sub-section 2.3 we explain the timefrequency (TF) representation method employed in this study. Finally, the characterization and reduction of motion artifacts in HRV spectrogram is explained in 2.4 and 2.5.

\subsection{Database and Experiment}

We used three sets of data in our experiment recorded throughout different exercises. 
1. A maximal running test from 11 healthy subjects who were recreational runners with a long background in running, conducted on a treadmill. A commercial three-channel ECG chest strap (Faros 360, Bittium Co., Finland) with the sampling frequency of $1 \mathrm{kHz}$ was used to monitor the cardiac electrical activity. The device simultaneously recorded a three-channel ACC signal at $100 \mathrm{~Hz}$ sampling frequency. Additionally, a spirometer recorded the oxygen consumption and respiratory frequency $(\mathrm{RF})$. The protocol of the experiment is sketched in Figure 1. There are consecutive running intervals lasting for three minutes followed by a pause (15-30 seconds) for a measurement. The protocol starts with the baseline measurement (stage I: standing) and the treadmill is switched on (females starting load at seven and males at eight $\mathrm{km} \mathrm{h}^{-1}$ ) from stage II. Then, the speed was incremented by $1 \mathrm{~km} \mathrm{~h}^{-1}$ and the running is continued up to the subject's exhaustion and his/her termination request. We performed the analysis on the last two minutes of each stage excluding the pause, assuming that the beginning of each stage might be unsteady, following the measurement pause. A sample set of our data of interest is highlighted in Figure 2.

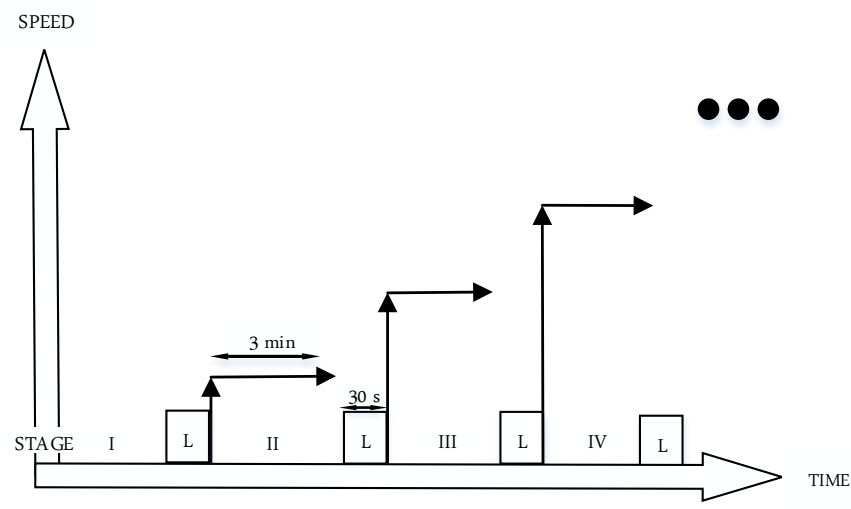

Figure 1: The maximal running test protocol. The protocol is initiated by a baseline stage (I). The running is then started for intervals of three minutes (stage II, III and ...). After each stage, the treadmill was paused for a measurement, indicated by $\mathrm{L}$ in the figure.

2. A moderate intensity level cycling test on a cycle ergometer from 84 subjects for four minutes with a constant load and pace. Similar configuration and the placement of sensors as the maximal running test were deployed. A Polar H10-based (Polar Electro, Kempele, Finland) prototype which was modified to produce one-lead ECG was used to record the reference ECG signal. The device also measures three-channel ACC signal simultaneously. A commercially available spirometer, MetaMax 3B (Cortex Biophysik $\mathrm{GmbH}$, Leipzig, Germany) was used for the respiratory measurements.

3. A gait walking test with the same device specification as the cycling on the same 84 subjects for four minutes with a constant pace.

We up-sampled the raw ECG signal in cycling and walking test to $1 \mathrm{kHz}$ before any further analysis for the sake of consistency of parameters with the running protocol and HRV analysis. All the participants were instructed beforehand on the test procedure and forbidden food and drinks (prior the test). A written consent was obtained from each of them. Table 1 summarizes the general characteristics of the study population. Before the experiment, an experienced physician checked up the subjects' cardiac status.

\subsection{Signal Construction}

\subsubsection{ECG processing}

We detect the ECG R-waves using Pan-Tompkins method [23]. The IBI signal is constructed and the ectopic beats are detected and edited by the method described in [2]. Then we use the time-varying integral pulse frequency modulation (TVIPFM) model proposed in [4], which presumes the instantaneous heart rate as a time-variant DC component, and a zero-mean modulating signal, expressed as $d_{H R M}(t)$ and $m(t)$, respectively. The idea of TVIPFM is based on the hypothesis that influence of ANS on sinoatrial (SA) node can be indicated by 

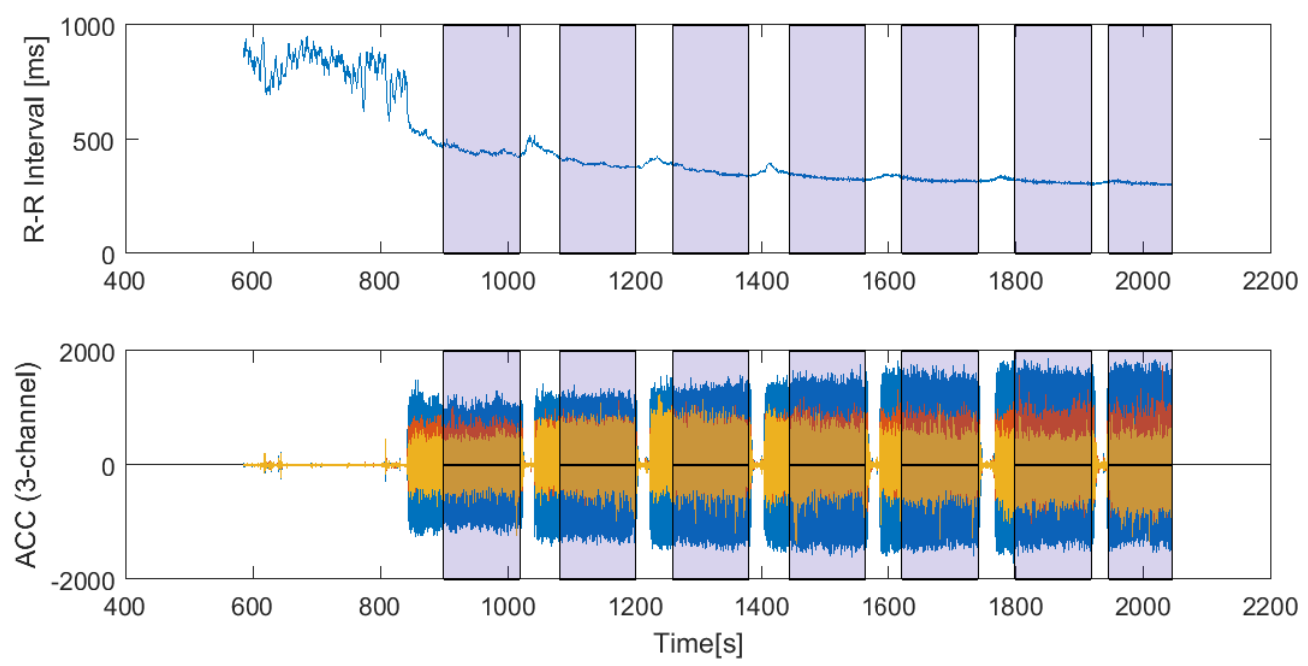

Figure 2: A sample RRI signal plus three-channel ACC signal (zero-mean) of the maximal running test is depicted in the first and second sub-figure. Blue, red and yellow color are respectively $x, y$ and $z$ channels of ACC. The highlight indicates our analysis segments (last two minutes of each stage). As it shows, the RRI decreases gradually, and reversely the ACC amplitude rises by the exercise workload increase. In some cases the subjects requested to terminate the protocol in the middle of the last workload and accordingly the last stage might be shorter than others, while, we utilize the whole data in those stages.

Table 1: The physiological characteristics of the subjects.

\begin{tabular}{ccc}
\hline Charactristic & \multicolumn{2}{c}{ Value } \\
\hline Data Set & Maximal Running Test & Cycling \& Walking \\
\hline Gender & $7 \mathrm{M}, 4 \mathrm{~F}$ & $44 \mathrm{M}, 40 \mathrm{~F}$ \\
Age $($ years $)$ & $37.1 \pm 6.5$ & $38.3 \pm 11.2$ \\
Height $(\mathrm{cm})$ & $173.8 \pm 7.8$ & $173.7 \pm 8.5$ \\
Mass $(\mathrm{kg})$ & $69.3 \pm 10.5$ & $74.9 \pm 17.9$ \\
BMI $\left(\mathrm{kg} \mathrm{m}^{-2}\right)$ & $22.8 \pm 2.1$ & $24.7 \pm 5.0$ \\
$V \mathrm{O}_{2} \max (\%)$ & $54.3 \pm 6.7$ & $46.0 \pm 12.4$ \\
\hline
\end{tabular}

$m(t)$ and a trigger is generated as the integral of $1+m(t)$ reaches to $d_{H R M}(t)$. The signal represented as $d_{H R M}(t)$ is basically time-varying local mean heart rate, which is the response to incremental physical stress-test and $m(t)$ is assumed to reflect the ANS activity of the cardiac system. TVIPFM model and the separation of the HRV components suppress the non-stationary influence of stress test. We conduct our analysis on the interpolated $m(t)$ by a cubic spline model of eight $\mathrm{Hz}$ sampling frequency.

HRV is naturally irregularly sampled at the R-peak time instants. By standard interpolation to a constant sampling rate the sampling theorem helps us to see that HRV modulation (by e.g. the cadence/pedaling and other motion components) at a frequency greater than the intrinsic Nyquist frequency of the heart $\left(\frac{d_{H R M}}{2}(\mathrm{~Hz})\right)$ will cause aliasing. The modulating energy beyond $\frac{d_{H R M}}{2}(\mathrm{~Hz})$ folds back to the interval of $\left[0(\mathrm{~Hz}), \frac{d_{H R M}}{2}(\mathrm{~Hz})\right][3]$.

\subsubsection{ACC processing}

The movement of participants is recorded by a three-channel ACC embedded on the ECG device and placed on the chest. It observes the subject's movement trajectory with three orthogonal channels from the same outlook as we monitor the heart. We assume that the ACC signal is a primary source to characterize the motion artifacts in HRV. The ACC signal provides the cadence/pedaling frequency and individual running/cycling pattern, as well as the movement profile in other directions, which their influence on HRV is overlooked in the literature.

To perceive the exerted movement artifact to the HRV analysis, we initially zero-mean the ACC signals and interpolate them to find values corresponding to the R-peak time instants 

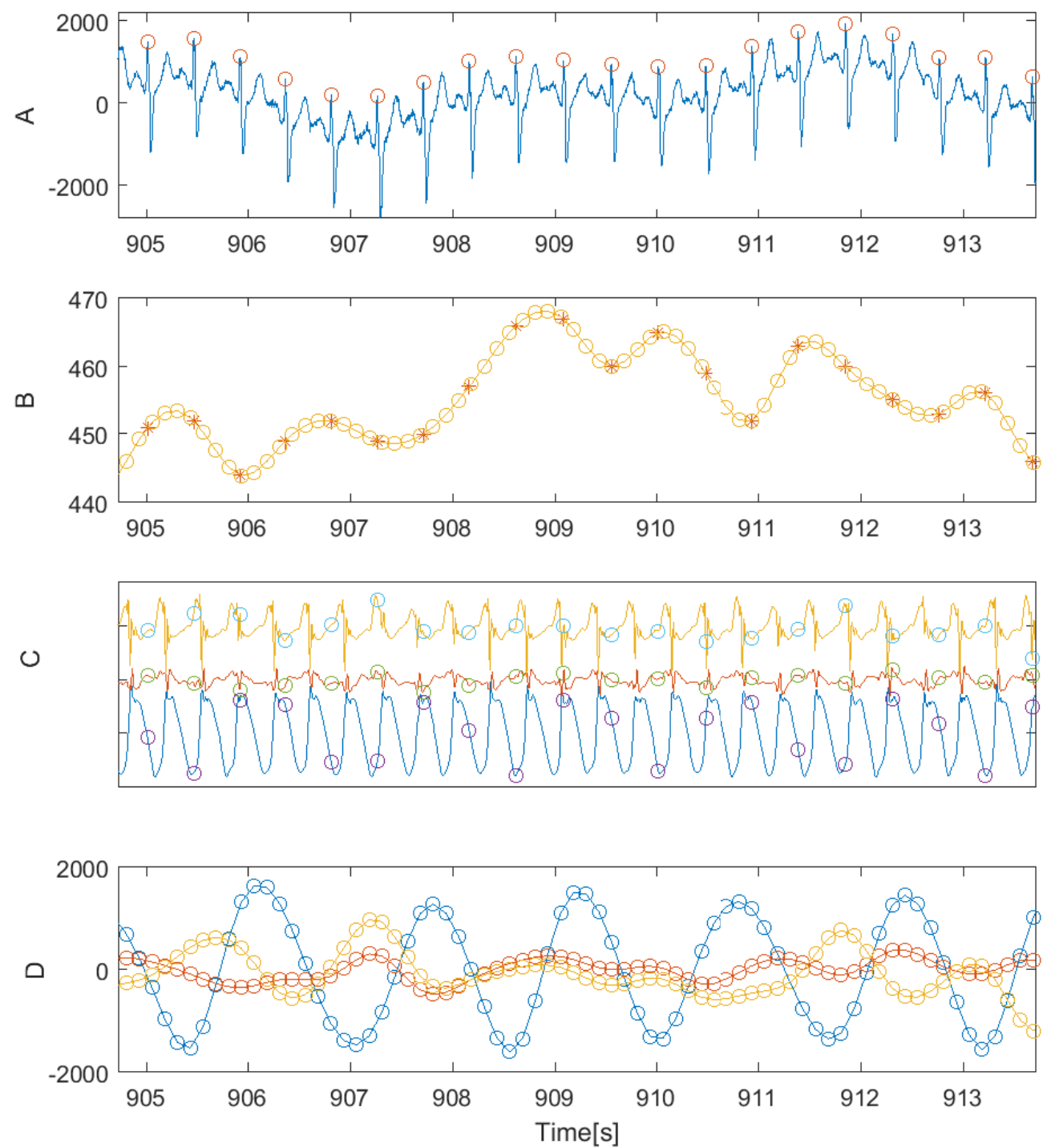

Figure 3: This figure shows the procedure of ACC preprocessing. Sub-figure A shows ECG signal and detected R peaks. The RRI time series and its interpolation is depicted in sub-figure B by red star and yellow circle, respectively. Sub-figure C shows three-channel ACC signals and the points taken at R-peak time instants are marked by circles. The interpolation of derived points from the ACC signals is expressed as $A C C_{R}(t)$ and is depicted in sub-figure $\mathrm{D}$.

and construct a time series with same number of samples as R-peaks. A portion of signal that underwent the procedure, is depicted in Figure 3. In essence, this interpolation of original ACC signal in the time-domain folds the HF components of ACC into HRV spectrum range. We express the constructed time series as $A C C_{R}(t)$, resembling the ACC values interpolated at Rpeak time instants. $A C C_{R}(t)$ portrays the impact of movement profile folded to the frequency range of $\mathrm{HRV}\left(\left[0(\mathrm{~Hz}), \frac{d_{H R M}}{2}(\mathrm{~Hz})\right]\right)$, as the effect of aliasing (illustrated in sub-figure $\mathrm{C}$ of Figure 3). The spectral pattern of $A C C_{R}(t)$ partially exists in the HRV spectrogram. Similar to $m(t), A C C_{R}(t)$ is interpolated by an eight $\mathrm{Hz}$ cubic spline function.

\subsection{Time-Frequency Representation}

There are variety of time-frequency methods to estimate the spectral content of a signal. In our study, we employ the smoothed pseudo Wigner-Ville distribution (SPWVD) model [19], 
which offers a high time and frequency resolution and has been largely used for the analysis of non-stationary biosignals [4, 3, 13]. SPWVD belongs to the Cohen's class of bilinear timefrequency representations. Considering $x(n)$ as a discrete signal, the SPWVD of $x(n)$ can be computed by (1),

$$
X(n, m)=2 \sum_{k=-N+1}^{N-1}|f(k)|^{2} \sum_{p=-M+1}^{M-1} t(p) r_{x}(n+p, k) e^{\frac{-2 \pi j k m}{N}},
$$

in which $r_{x}(n, k)$ is the instantaneous autocorrelation function, $n$ and $m$ are the time and frequency indices. $f(k)$ and $t(p)$ are the frequency and time smoothing normed window, sized $2 N-1$ and $2 M-1$, respectively. Figure 4 shows TF representation of a sample $m(t)$ and $A C C_{R}(t)$ computed by the SPWVD model in logarithmic scale.
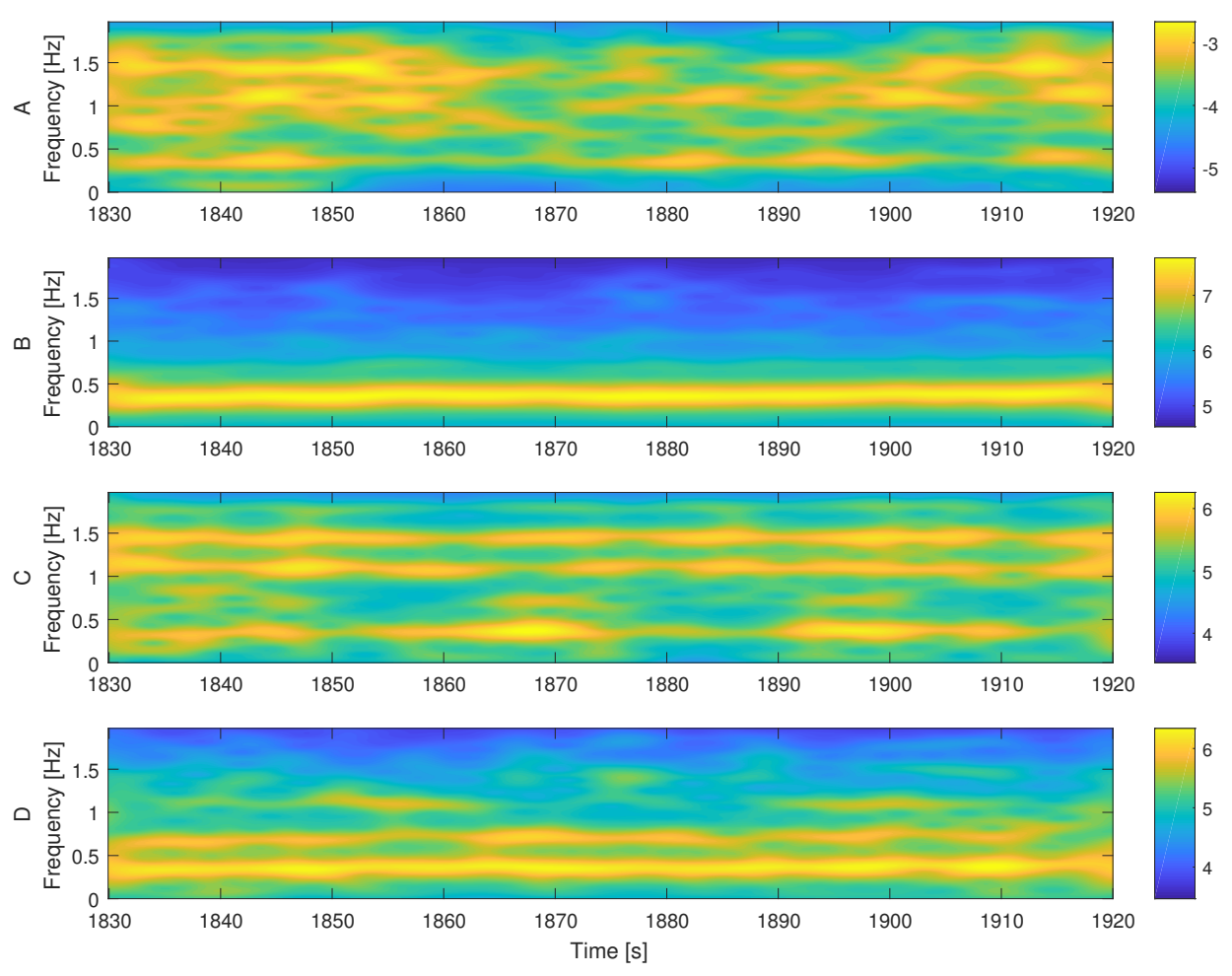

Figure 4: The TF representation of a sample $m(t)$ is depicted in sub-figure A, and three channels of $A C C_{R}(t)$ are depicted in sub-figures $\mathrm{B}, \mathrm{C}$ and $\mathrm{D}$. All spectrograms power values are in logarithmic scale. Shared frequency components amongst $A C C_{R}(t)$ and $m(t)$ spectrograms is visible (e.g. the consistent component of $A C C_{R}^{x}(t)$ in sub-figure B situated at $0.4 \mathrm{~Hz}$ exists with variable energy in $m(t)$ spectrogram.).

\subsection{Characterization of Frequency Components}

The classical power spectrum of HRV is divided into two main components, including LF component spanning within $[0.04 \mathrm{~Hz}, 0.15 \mathrm{~Hz}]$, which considers to be an indicator of parasympathetic and sympathetic activity of ANS, and $\mathrm{HF}$ component ranging within $[0.15 \mathrm{~Hz}, 0.4 \mathrm{~Hz}]$, considered as a measure of parasympathetic activity of ANS, dominated mainly by the respiratory sinus arrhythmia (RSA) [6]. However, during exercise by the rise of RF, the HRV HF upper range is redefined to the intrinsic Nyquist frequency of the heart and thus the meaningful HF component is accommodated within $\left[0.15(\mathrm{~Hz}), \frac{d_{H R M}}{2}(\mathrm{~Hz})\right][3,21]$.

Similar to the literature $[3,13,5]$, we encounter homogeneous frequency components corresponding to the cadence/pedaling frequency between $A C C_{R}(t)$ and $m(t)$ spectrograms (those might be aliased/not-aliased patterns). In sub-figure B of Figure 4, the $A C C_{R}^{x}(t)$ spectrogram owns a narrow energy band at approximately $0.4 \mathrm{~Hz}$ corresponding to the aliased cadence 
frequency. Likewise throughout the exercise, a component at the very same frequency of $m(t)$ spectrogram (sub-figure A) can be seen, while the the strength is not as consistent as the $A C C_{R}^{x}(t)$ spectrogram. Apart from cadence/pedaling, there are components associated with other movement trajectories predominantly in $A C C_{R}^{y}(t)$ and $A C C_{R}^{z}(t)$ spectrograms which can be perceived in $m(t)$ spectrogram.

Figure 4 illustrates that joint components co-exist in both HRV and ACC signals. However, it should be noted that the ACC sensor channels might record partially correlated movement trajectories due to the placement of the sensor, and hence there might be similar patterns in all three channels of $A C C_{R}(t)$, which is the case in Figure 4. We aim to suppress the joint components co-existing in the spectrograms of $A C C_{R}(t)$ and $m(t)$, which reduces the motion artifact components modulated over HRV. The reduction method should handle these two issues:

1. The components labeled as motion influence in $m(t)$ spectrogram possess dissimilar strength and bandwidth at each time instant, and

2. cadence/pedaling-related aliased constituents are not the only disturbance of $m(t)$.

There is a variability in strength and bandwidth of the proportion of motion influence in HRV spectrograms, as shown in Figure 4 and one can not presume a uniform motion disturbance in the filtering solution for the problem. In addition, there are also joint components independent of the cadence/pedaling frequency as shown in sub-figure $\mathrm{C}$ of Figure 4 which should be taken into account. So despite the correlation between different channels of ACC, all of those should be part of the filtering approach. Accordingly to effectively reduce the disturbance from $m(t)$ spectrogram, we propose a filtering approach in which all three $A C C_{R}(t)$ spectrograms at each time instant are taken into account. The following sub-section outlines the procedure.

\subsection{Reduction of Motion Influence from HRV Spectrogram}

Our approach to utilize the ACC signal in HRV analysis is the following. We basically assume that the $m(t)$ spectrogram consists of a mixture of the $A C C_{R}(t)$ spectrograms and also the actual "intrinsic" HRV and some noise, and the problem is to estimate how much each of those is seen in the mixture. To reduce the complexity, we only consider linear mixtures in which case we can utilize coding theory and formulate the task as an error correcting problem. The channel decoding problem used in this paper tries to recreate the target signal (here the HRV spectrum) from multiple sources that affect it (here the different acceleration directions in spectral domain) as well as possible. Basically, trying to sense the direction(s) and magnitude(s) of the acceleration effect on HRV with the model weights.

Thus, for each time instant $t_{i}$, let $M=\left[S_{m}\left(t_{i}, f_{1}\right) \cdots S_{m}\left(t_{i}, f_{N}\right)\right]^{T} \in \mathbb{R}^{N}$ be the observed $m(t)$ spectrum in $N$ frequency bins. In addition, let us combine the ACC spectra at that instant into the matrix $A=\left[\Omega_{x} \Omega_{y} \Omega_{z}\right]$ where the individual channel spectra are $\Omega_{(.)}=$ $\left[S_{(.)}\left(t_{i}, f_{1}\right) \cdots S_{(.)}\left(t_{i}, f_{N}\right)\right]^{T} \in \mathbb{R}^{N}$ and (.) is just a placekeeper for the $A C C_{R}(t)$ channel x, y or $\mathrm{z}$. We can now formulate the $l_{1}$ channel decoding problem

$$
\min _{\alpha \in \mathbb{R}^{3}}\|M-A \alpha\|_{1},
$$

where we wish to recover the mixture coefficients $\alpha$ from the "corrupted" measurements $M=A \alpha+e$, where the noise $e$ contains the intrinsic HRV control effects as well as random noise. So, the ACC spectra are considered, in essence, to be corrupted by the actual HRV, and under suitable conditions the mixture coefficients are the unique solution of (2) [7]. In this paper, $l_{1}$-norm is used that has the rather unique property of favouring sparse solutions, i.e. ones with large spanning very well fitting parts at the same time allowing for larger variations in other more localized parts compared to e.g. $l_{2}$-norm that penalizes any large deviation rather heavily no matter how localized (in the spectrum in our case).

The minimization process is repeated for each time instant $t_{i}$, and finally the optimal coefficients can be used to reduce the influence of $A C C_{R}(t) \mathrm{s}$ and acquire the residuals $r=$ $M-A \alpha$ which constitute, in every important respect, the filtered spectrogram in which the HRV components explained by the acceleration have been diminished. This time-independent attenuation procedure in which we apply to each column of spectrograms avoids non-stationary influences e.g. varying respiratory frequency. Figure 5 illustrates the $A C C_{R}(t)$ spectrograms and their corresponding optimal mixture coefficients $(\alpha)$ in time. In the residual $r$, we are 


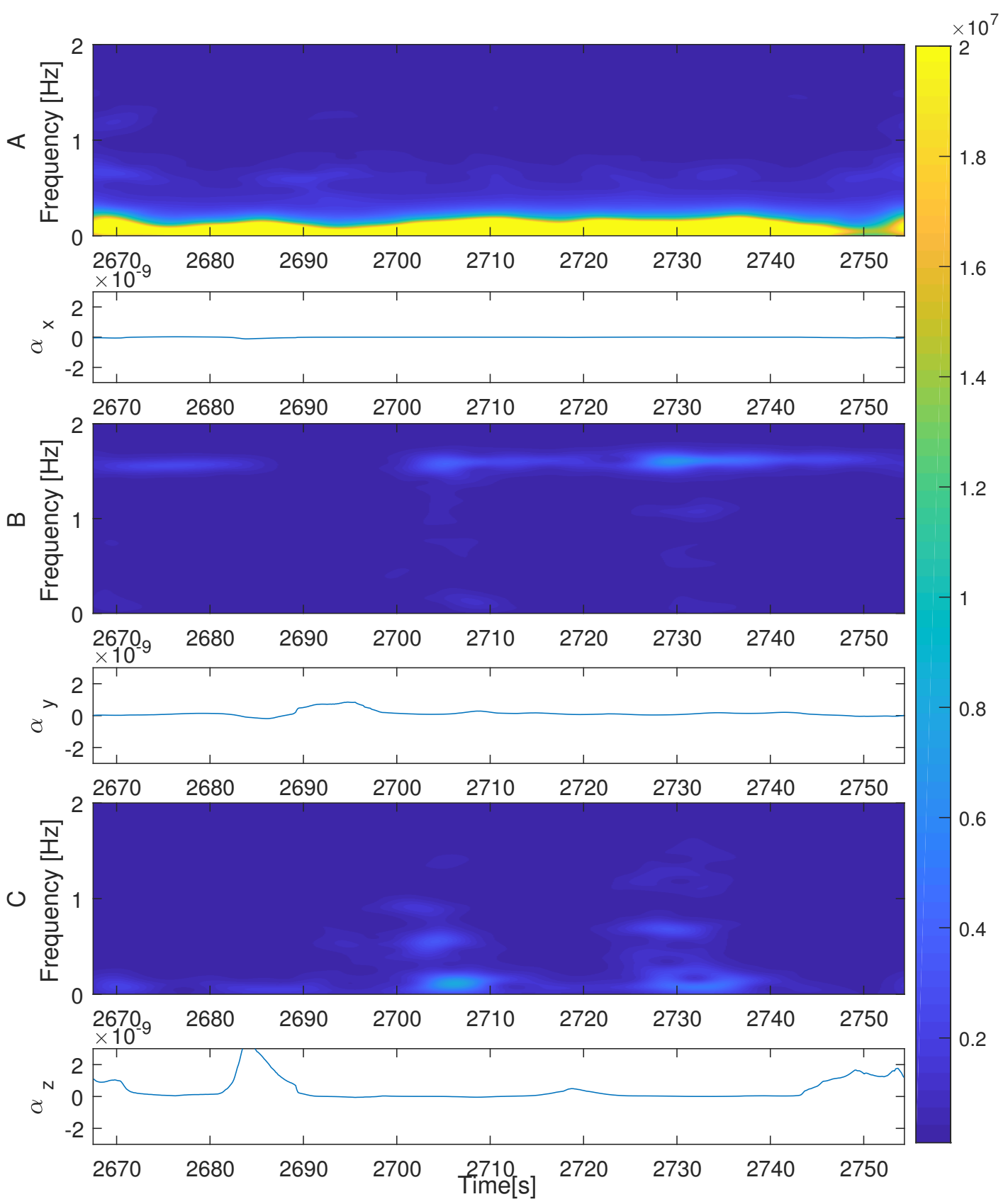

Figure 5: $A C C_{R}(t)$ spectrograms and their coefficient signals. These three spectrograms are timefrequency representation of $A C C_{R}^{i}(t)\{i: x, y, z\}$ channels from top to bottom, respectively as well as their corresponding mixture coefficient.

mainly interested in the positive part

$$
r_{+}= \begin{cases}r & \text { if } r \geq 0 \\ 0 & \text { if } r<1\end{cases}
$$

In the spectral domain, the ACC signals and the HRV during exercise clearly share power at several common frequencies (that we are trying to filter out) and also share parts where there is only negligible power. These together form the large spanning similar parts of the spectra which can be fitted together using the $l_{1}$-norm simultaneously disregarding energy at other frequencies not common. The power at these other frequencies, however, alter the time domain the shape/form of the signals making the time domain matching less likely with the similar decoding approach and would likely require different kind of a signal model.

since the power spectra are non-negative functions of the frequency, and the residual is negative if and only if the subtraction of the mixture tries to remove more power than was 


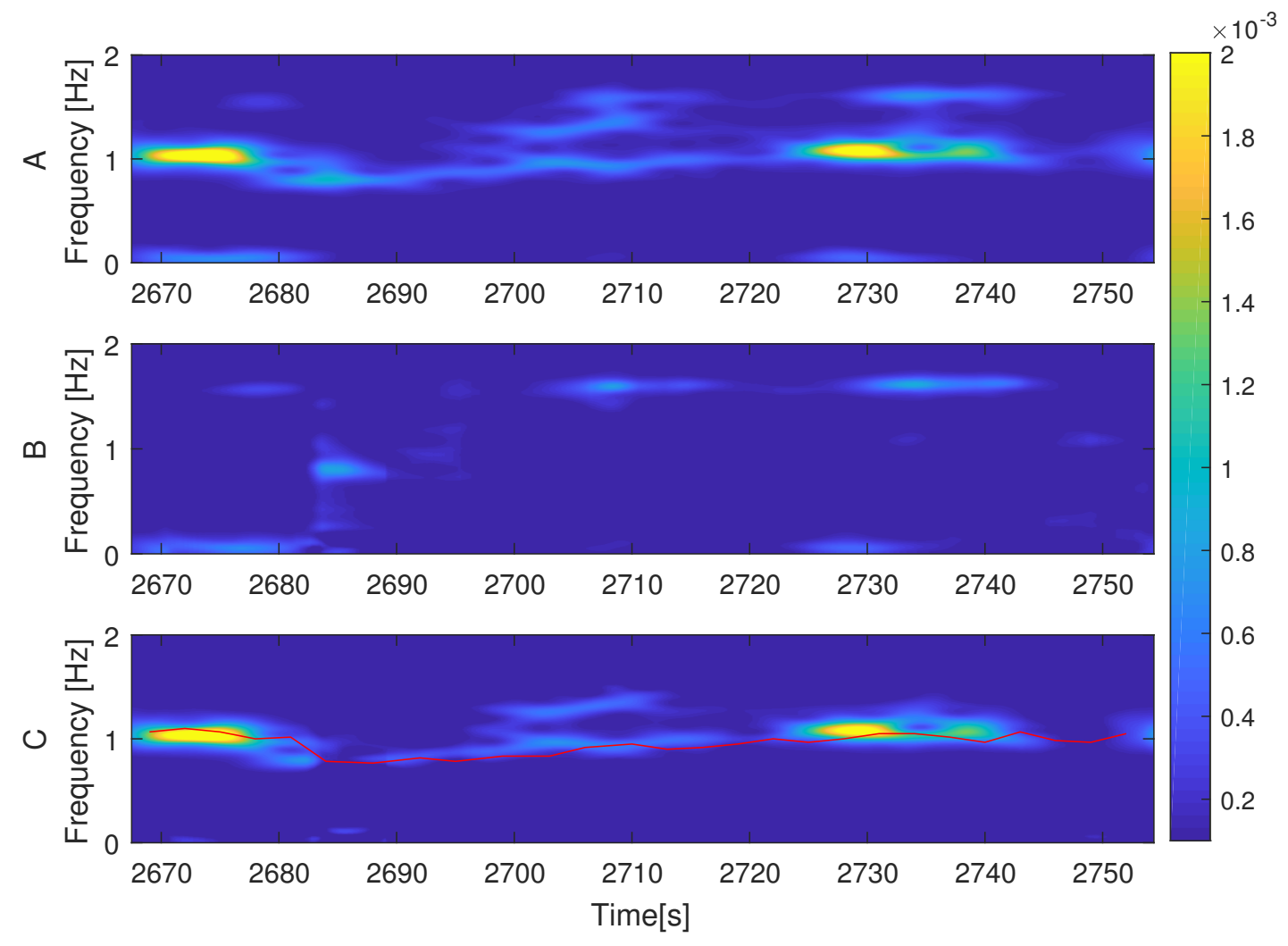

Figure 6: original $m(t)$ spectrogram is depicted in sub-figure A. Sub-figure B is the mixture matrix computed by $A C C_{R}(t)$ spectrograms and mixture coefficients $(\alpha)$ illustrated in Figure 5 . The residual spectrogram is visualized in sub-figure $\mathrm{C}$. The $\mathrm{RF}$ is also plotted in red, showing the remaining RSA component in the residual spectrogram. This sample case is from the latest stage of the running, after AT and the average HF energy of the original and residual spectrograms are $\overline{P_{H F}^{o r g}}=0.0715\left(s^{2}\right)$ and $\overline{P_{H F}^{r e s}}=0.0489\left(s^{2}\right)$, respectively, and $q=19.1 \%$.

present in the HRV spectrum to begin with. This interpretability is also one key factor why the $l_{1}$-norm is used in the minimization. The usage of the more conventional $l_{2}$-norm would, in practice, lead to less sparse residual with more overreductions of the mixture (negative residuals) as that norm basically trades the "overall fit" for reducing peak residual values. Also, when operating on the power spectra of certain length time windows, we are not using the phase information. This allows us not to consider the exact time delays of effects between the time domain signals, which is beneficial as in many cases it is the HRV spectrum and the distribution of power between low and high frequency parts of it that one is interested in as one of the main features used the HRV analysis.

The mixture is visualized in sub-figure B of Figure 6 and the residual matrix is depicted in sub-figure C. Evidently the mutual components of $A C C_{R}(t)$ s and $m(t)$ are attenuated and the residual spectrogram owns less energy in connection with the motion trajectory. To quantify the amount of the motion influence over HRV, we define the index

$$
q=1-\frac{\left\|r_{+}\right\|_{1}}{\|M\|_{1}}
$$

Additionally, mean HF power of the original $\left(\overline{P_{H F}^{o r g}}\right)$ and residual $\left(\overline{P_{H F}^{r e s}}\right)$ spectrograms are calculated in the experiment, as the difference shows to what extent the spectrogram has been filtered.

\section{Results}

Table 2 describes the analysis results for walking and cycling and Table 3 for incremental running set. The average breathing $(\overline{R F})$ and heart rate $(\overline{H R})$ as well as the original $\left(\overline{P_{H F}^{\text {org }}}\right)$ 
and residual $\left(\overline{P_{H F}^{r e s}}\right)$ mean HF power of HRV and $q$ index is stated. Table 2 is the average values acquired from 84 subjects throughout the walking and cycling protocols. However, Table 3 offers stage-by-stage values and the number of available data in the corresponding segment (columns labeled as $\mathrm{n}$ ). As described in subsection 2.1, in a maximal running test, the subjects accomplish variable number of stages based on their physical strength and for that reason the $\mathrm{n}$ decreases gradually in Table 3. In addition, the data quality of some segments were not appropriate which were excluded from the analysis. The HRV spectrogram is substantially influenced by the pedaling, cadence and characterized motion artifacts, ranging from a few percent to more than $60 \%$ at some cases computed by the $q$ index. The HF energy of the original spectrogram might drop significantly in the computed residual spectrograms. To illustrate the trend of motion influence throughout different stages of the running test Figure 7 shows the mean $q$ index, high-frequency power of the original and residual signal at each stage of the running protocol. The rise of average $q$ index at the initial stages of the protocol in sub-figure A can be justified by workload increment, while it remains steady in the next two levels. From that workload some of the participants start to terminate their exercise and a couple of participants with high fitness level accomplished the remaining five stages. Subfigure B shows that throughout different exercise stages, approximately half the HF power is filtered, which affirms high level of motion influence in HRV spectrum.

Table 2: The results acquired from the cycling and walking test set

\begin{tabular}{ccc}
\hline & \multicolumn{2}{c}{ Data Set } \\
\hline Indicator (unit) & Cycling & Walking \\
\hline$\overline{R F}(\mathrm{bpm})$ & $25.5 \pm 4.2$ & $31.8 \pm 7.2$ \\
\hline$\overline{H R}(\mathrm{bpm})$ & $121.5 \pm 15.3$ & $136.5 \pm 18.6$ \\
\hline$\overline{P_{H F}^{\text {org }}\left(s^{2}\right)}$ & $0.1238 \pm 0.1139$ & $0.5753 \pm 0.3792$ \\
\hline$\overline{P_{H F}^{r e s}}\left(s^{2}\right)$ & $0.0376 \pm 0.0311$ & $0.1391 \pm 0.0898$ \\
\hline$q(\%)$ & $38.8 \pm 17.3$ & $32.00 \pm 16.73$ \\
\hline
\end{tabular}

Table 3: The results acquired from the cycling and walking test set

\begin{tabular}{lcccccc}
\hline Indicator (unit) & $\overline{R F}(\mathrm{bpm})$ & $\overline{H R}(\mathrm{bpm})$ & $\overline{P_{H F}^{\text {org }}}\left(s^{2}\right)$ & $\overline{P_{H F}^{\text {res }}}\left(s^{2}\right)$ & $q(\%)$ & $\mathrm{n}$ \\
\hline Stage II & $25.4 \pm 4.2$ & $121.8 \pm 10.9$ & $0.1468 \pm 0.1348$ & $0.0601 \pm 0.0585$ & $20.71 \pm 14.35$ & 11 \\
\hline Stage III & $27.8 \pm 4.2$ & $130.9 \pm 14.0$ & $0.1095 \pm 0.0624$ & $0.0335 \pm 0.0269$ & $31.40 \pm 17.47$ & 11 \\
\hline Stage IV & $30.7 \pm 6.6$ & $141.9 \pm 15.3$ & $0.1077 \pm 0.0659$ & $0.0299 \pm 0.0236$ & $43.91 \pm 11.33$ & 11 \\
\hline Stage V & $33.0 \pm 7.3$ & $150.0 \pm 15.34$ & $0.1054 \pm 0.0688$ & $0.0324 \pm 0.0289$ & $44.27 \pm 12.47$ & 11 \\
\hline Stage VI & $36.1 \pm 6.9$ & $158.7 \pm 14.0$ & $0.1166 \pm 0.0920$ & $0.0422 \pm 0.0381$ & $42.60 \pm 15.02$ & 11 \\
\hline Stage VII & $38.6 \pm 6.6$ & $166.0 \pm 13.0$ & $0.1324 \pm 0.1029$ & $0.0524 \pm 0.0466$ & $34.46 \pm 13.15$ & 11 \\
\hline Stage VIII & $40.6 \pm 6.5$ & $168.0 \pm 10.0$ & $0.1215 \pm 0.1498$ & $0.0475 \pm 0.0580$ & $33.83 \pm 13.77$ & 8 \\
\hline Stage IX & $42.0 \pm 6.3$ & $162.8 \pm 9.8$ & $0.1351 \pm 0.1878$ & $0.0525 \pm 0.0660$ & $27.28 \pm 2.85$ & 5 \\
\hline Stage X & $45.0 \pm 6.0$ & $182.3 \pm 3.0$ & $0.2320 \pm 0.3446$ & $0.0831 \pm 0.1145$ & $35.96 \pm 14.62$ & 3 \\
\hline Stage XI & $51.6 \pm 6.8$ & $186.0 \pm 2.6$ & $0.3655 \pm 0.5339$ & $0.1067 \pm 0.1235$ & $41.80 \pm 28.39$ & 3 \\
\hline Stage XII & 48 & 187 & 0.0585 & 0.0337 & 27.60 & 1 \\
\hline
\end{tabular}

\section{Conclusion and Future Works}

By the rise of wearable technologies there is a substantial demand for the extraction of physiological information such as HRV from nowadays easy-to-measure biomarkers. However, the reliability of the information throughout different intensity levels of exercise is under question. In this study we evaluated the influence of motion artifacts and subject's movement trajectory recorded by ACC over HRV throughout a walking, cycling and a maximal running test. We proposed a filtering approach in which we employ the ACC signals to attenuate the effect of joint components from the HRV spectrogram by means of an error correcting problem formulation and $l_{1}$ channel decoding problem. The quantitative result shows a substantial influence of motion on HRV during three different exercise protocols (in agreement with [12, 3, 5]), and underlines the necessity of HRV filtering for a more precise interpretation of HRV indexes and underlying physiology. In our method we took into account not only the cadence/pedaling as a major disturbance, but also other components in the motion trajectory of the athletes. Our methodology does not assume a constant bandwidth around the characterized motion 

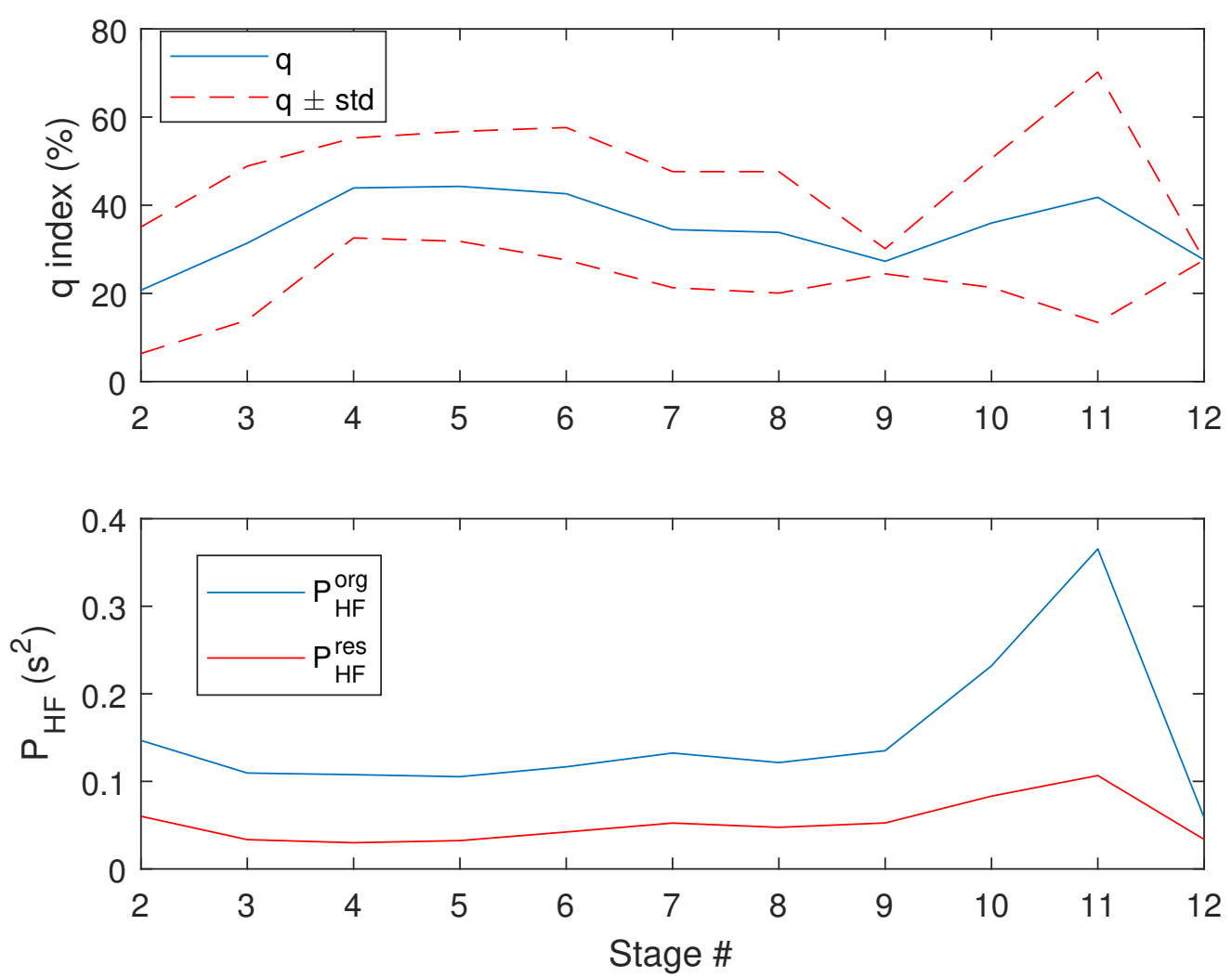

Figure 7: The average $q$ index and its variation at each workload of the running test is depicted in sub-figure A. Sub-figure B shows the average original and residual HF power in blue and red lines at each workload, respectively.

components and also uniform influence in time, but instead at each time instant computes and suppress the components which can be described by the inertial signals from the HRV spectrogram.

Considering the rising heart rate throughout exercise, at some times the motion components might coincide at the same frequency with the RSA components of HRV. The origin/proportion of energy bands where motion components and RSA overlaps is not easily distinguishable. As a limitation of this study, we neglected the RSA component's overlap with the motion and hence the RSA might be affected at some time instants in the residual matrix by our filtering pipeline. It might also affect the computed indexes or immoderate values at some stages in Tables 2 and 3 , while study of this issue is in our future work plan. 


\section{References}

[1] U. R. Acharya, K. P. Joseph, N. Kannathal, C. M. Lim, and J. S. Suri. Heart rate variability: a review. Medical and biological engineering and computing, 44(12):10311051, 2006.

[2] I. Alikhani, K. Noponen, A. Hautala, R. Ammann, and T. Seppänen. Spectral data fusion for robust ecg-derived respiration with experiments in different physical activity levels. In Proceedings of the 10th International Joint Conference on Biomedical Engineering Systems and Technologies - Volume 5: HEALTHINF, (BIOSTEC 2017), pages 88-95. INSTICC, SciTePress, 2017.

[3] R. Bailón, N. Garatachea, I. de la Iglesia, J. A. Casajús, and P. Laguna. Influence of running stride frequency in heart rate variability analysis during treadmill exercise testing. IEEE transactions on biomedical engineering, 60(7):1796-1805, 2013.

[4] R. Bailón, G. Laouini, C. Grao, M. Orini, P. Laguna, and O. Meste. The integral pulse frequency modulation model with time-varying threshold: application to heart rate variability analysis during exercise stress testing. IEEE transactions on biomedical engineering, 58(3):642-652, 2011

[5] G. Blain, O. Meste, A. Blain, and S. Bermon. Time-frequency analysis of heart rate variability reveals cardiolocomotor coupling during dynamic cycling exercise in humans. American Journal of Physiology-Heart and Circulatory Physiology, 296(5):H1651-H1659, 2009.

[6] A. J. Camm, M. Malik, J. Bigger, G. Breithardt, S. Cerutti, R. Cohen, P. Coumel, E. Fallen, H. Kennedy, R. Kleiger, et al. Heart rate variability: standards of measurement, physiological interpretation and clinical use. task force of the european society of cardiology and the north american society of pacing and electrophysiology. Circulation, 93(5):1043-1065, 1996.

[7] E. J. Candes and T. Tao. Decoding by linear programming. IEEE transactions on information theory, 51(12):4203-4215, 2005.

[8] R. M. Carney, J. A. Blumenthal, P. K. Stein, L. Watkins, D. Catellier, L. F. Berkman, S. M. Czajkowski, C. O'connor, P. H. Stone, and K. E. Freedland. Depression, heart rate variability, and acute myocardial infarction. Circulation, 104(17):2024-2028, 2001.

[9] F. Cottin, C. Médigue, P.-M. Leprêtre, Y. Papelier, J.-P. Koralsztein, and V. Billat. Heart rate variability during exercise performed below and above ventilatory threshold. Medicine $\&$ Science in Sports \& Exercise, 36(4):594-600, 2004.

[10] F. Cottin, C. Médigue, P. Lopes, P.-M. Leprêtre, R. Heubert, and V. Billat. Ventilatory thresholds assessment from heart rate variability during an incremental exhaustive running test. International journal of sports medicine, 28(04):287-294, 2007.

[11] J. M. Dekker, R. S. Crow, A. R. Folsom, P. J. Hannan, D. Liao, C. A. Swenne, and E. G. Schouten. Low heart rate variability in a 2-minute rhythm strip predicts risk of coronary heart disease and mortality from several causes. Circulation, 102(11):1239-1244, 2000.

[12] A. J. Hautala, T. H. Mäkikallio, T. Seppänen, H. V. Huikuri, and M. P. Tulppo. Shortterm correlation properties of $\mathrm{r}-\mathrm{r}$ interval dynamics at different exercise intensity levels. Clinical physiology and functional imaging, 23(4):215-223, 2003.

[13] D. Hernando, A. Hernando, J. A. Casajús, P. Laguna, N. Garatachea, and R. Bailón. Methodological framework for heart rate variability analysis during exercise: application to running and cycling stress testing. Medical $\mathcal{E}$ biological engineering $\mathcal{E}$ computing, pages $1-14,2017$.

[14] D. Jarchi and A. J. Casson. Description of a database containing wrist ppg signals recorded during physical exercise with both accelerometer and gyroscope measures of motion. Data, 2(1):1, 2016.

[15] R. E. Kleiger, P. K. Stein, and J. T. Bigger. Heart rate variability: measurement and clinical utility. Annals of Noninvasive Electrocardiology, 10(1):88-101, 2005.

[16] M. T. La Rovere, J. T. Bigger, F. I. Marcus, A. Mortara, P. J. Schwartz, A. A. Tone, R. A. M. I. Investigators, et al. Baroreflex sensitivity and heart-rate variability in prediction of total cardiac mortality after myocardial infarction. The Lancet, 351(9101):478-484, 1998.

[17] H. C. Lunt, J. Corbett, M. J. Barwood, and M. J. Tipton. Cycling cadence affects heart rate variability. Physiological measurement, 32(8):1133, 2011. 
[18] V. Magagnin, T. Bassani, V. Bari, M. Turiel, R. Maestri, G. D. Pinna, and A. Porta. Non-stationarities significantly distort short-term spectral, symbolic and entropy heart rate variability indices. Physiological measurement, 32(11):1775, 2011.

[19] W. Martin and P. Flandrin. Wigner-ville spectral analysis of nonstationary processes. IEEE Transactions on Acoustics, Speech, and Signal Processing, 33(6):1461-1470, 1985.

[20] I. Mendia-Iztueta, K. Monahan, H. Kyröläinen, and E. Hynynen. Assessment of heart rate variability thresholds from incremental treadmill tests in five cross-country skiing techniques. PloS one, 11(1):e0145875, 2016.

[21] S. Michael, K. S. Graham, and G. M. Davis. Cardiac autonomic responses during exercise and post-exercise recovery using heart rate variability and systolic time intervals - a review. Frontiers in physiology, 8:301, 2017.

[22] L. Mourot, M. Bouhaddi, S. Perrey, S. Cappelle, M.-T. Henriet, J.-P. Wolf, J.-D. Rouillon, and J. Regnard. Decrease in heart rate variability with overtraining: assessment by the poincare plot analysis. Clinical physiology and functional imaging, 24(1):10-18, 2004.

[23] J. Pan and W. J. Tompkins. A real-time qrs detection algorithm. IEEE transactions on biomedical engineering, (3):230-236, 1985.

[24] M. P. Tulppo, T. Makikallio, T. Takala, T. Seppanen, and H. V. Huikuri. Quantitative beat-to-beat analysis of heart rate dynamics during exercise. American journal of physiology-heart and circulatory physiology, 271(1):H244-H252, 1996.

[25] M. P. Tulppo, T. H. Mäkikallio, T. Seppänen, R. T. Laukkanen, and H. V. Huikuri. Vagal modulation of heart rate during exercise: effects of age and physical fitness. American Journal of Physiology-Heart and Circulatory Physiology, 274(2):H424-H429, 1998.

[26] M. Weippert, K. Behrens, A. Rieger, R. Stoll, and S. Kreuzfeld. Heart rate variability and blood pressure during dynamic and static exercise at similar heart rate levels. PLoS One, 8(12):e83690, 2013. 